\title{
Pharmacokinetic Study of Osimertinib in Cancer Patients with Mild or Moderate Hepatic Impairment $\mathbf{s}^{\mathbb{\Omega}}$
}

\author{
Enrique Grande, R. Donald Harvey, Benoit You, Jaime Feliu Batlle, Hal Galbraith, \\ John Sarantopoulos, Suresh S. Ramalingam, Helen Mann, Karen So, Martin Johnson, \\ and Karthick Vishwanathan
}

\begin{abstract}
Medical Oncology Department, Ramón y Cajal Hospital, Madrid, Spain (E.G.); Department of Hematology and Medical Oncology, Emory University School of Medicine, Winship Cancer Institute, Atlanta, Georgia (R.D.H., S.S.R.); Medical Oncology, Faculté de Médecine Lyon-Sud, Université Claude Bernard Lyon-1, Institute de Cancérologie des Hospices Civils de Lyon, Lyon, France (B.Y.); Medical Oncology Department, La Paz University Hospital, Autonoma University of Madrid (affiliated with CIBERONCInstituto de Salud Carlos III), Madrid, Spain (J.F.B.); IQVIA, Kansas City, Missouri (H.G.); Institute for Drug Development, Mays Cancer Center at University of Texas, Health San Antonio, San Antonio, Texas (J.S.); Global Medicines Development, AstraZeneca, Cambridge, United Kingdom (H.M., K.S.); and Quantitative Clinical Pharmacology, Early Clinical Development IMED Biotech Unit, AstraZeneca, Cambridge, United Kingdom (M.J., K.V.)
\end{abstract}

Received December 21, 2018; accepted February 20, 2019

\section{ABSTRACT}

Osimertinib, an epidermal growth factor receptor (EGFR)-tyrosine kinase inhibitor (TKI), undergoes significant hepatic elimination. In this phase 1 study, we assessed the effects of mild and moderate hepatic impairment on the pharmacokinetics (PK) of osimertinib in patients with malignant solid tumors. In part $A$, patients with normal hepatic function, mild hepatic impairment, and moderate hepatic impairment, according to the Child-Pugh classification, received a single $80 \mathrm{mg}$ oral dose of osimertinib. Standard PK measures were assessed. In part $B$, patients could continue osimertinib treatment if deemed clinically appropriate. We compared these study results with a population PK analysis including other osimertinib clinical studies. Geometric mean osimertinib plasma concentrations were lower in patients with mild $(n=7)$ or moderate hepatic impairment $(n=5)$ versus normal hepatic function ( $n=10$ ): $C_{\max }$ was reduced to $51 \%$ and $61 \%$, respectively; area under the curve was reduced to $63 \%$ and $68 \%$, respectively. PK results for the metabolites were similar. No apparent differences in the safety profile were found between patients with normal hepatic function and patients with mild or moderate hepatic impairment. Comparison of these study results with National Cancer Institute-Organ Dysfunction Working Group criteria from population PK analysis showed osimertinib exposure was not affected by hepatic impairment. No dose adjustment is required for osimertinib when treating patients with mild or moderate hepatic impairment. No apparent differences in the safety of osimertinib were found between patients with normal hepatic function and mild or moderate hepatic impairment.

\section{Introduction}

Osimertinib is an oral central nervous system-active, third-generation, irreversible epidermal growth factor receptor (EGFR) tyrosine kinase inhibitor (TKI) selective for both EGFR-TKI-sensitizing (EGFRm) and EGFR T790M resistance mutations (Cross et al., 2014; Mok et al., 2017;

This study (NCT02161770) was sponsored by AstraZeneca at the Institute for Drug Development, Cancer Therapy and Research Center, University of Texas Health Center San Antonio; this work was supported by the Cancer Center Support [Grant P30CA054174] to J.S.

https://doi.org/10.1124/jpet.118.255919.

S This article has supplemental material available at jpet.aspetjournals.org.
Goss et al., 2018; Soria et al., 2018). Osimertinib is approved in the European Union and the United States as a first-line treatment of patients with EGFRm metastatic non-small cell lung cancer (NSCLC) and for patients with T790M-positive metastatic NSCLC after disease progression on an EGFR-TKI (https://www.accessdata.fda.gov/ drugsatfda_docs/label/2018/208065s008lbl.pdf; Socinski et al., 2018).

In the AURA study (phase 1 component), osimertinib displayed dose-proportional increases in exposure from 20 to $240 \mathrm{mg} /$ day and had a mean half-life of 48.3 hours; steady state was achieved after 15 days of daily dosing (Planchard et al., 2016). The linear pharmacokinetics (PK) of osimertinib

ABBREVIATIONS: AE, adverse event; AST, aspartate aminotransferase; AUC, area under the plasma-concentration time curve from zero to infinity; AUCss, area under the plasma concentration time curve at steady state; AZ5104, $\mathrm{N}$-[2-[2-(dimethylamino)ethyl-methylamino]-5-[[4-(1Hindol-3-yl)pyrimidin-2-yl]amino]-4-methoxyphenyl]prop-2-enamide; AZ7550, N-[4-methoxy-5-[[4-(1-methylindol-3-yl)pyrimidin-2-yl]amino]-2[methyl-[2-(methylamino)ethyl]amino]phenyl]prop-2-enamide; Cl, confidence interval; CL/F, apparent plasma clearance; EGFR, epidermal growth factor receptor; EGFRm, EGFR-TKI sensitizing mutations; FDA, Food and Drug Administration; LOQ, limit of quantification; NCI-ODWG, National Cancer Institute Organ Dysfunction Working Group; NSCLC, non-small cell lung cancer; PK, pharmacokinetics; SAE, serious adverse event; $t_{1 / 2 \lambda z}$, terminal half-life; TKI, tyrosine kinase inhibitor; $t_{\max }$, time of maximum concentration; ULN, upper limit of normal; Vz/F, apparent volume of distribution. 
suggest that the effect of a single dose is likely similar to steady state.

In vitro, osimertinib and its pharmacologically active metabolites [AZ5104 (N-[2-[2-(dimethylamino)ethyl-methylamino]-5-[[4( $1 H$-indol-3-yl)pyrimidin-2-yl]amino]-4-methoxyphenyl]prop2-enamide) and AZ7550 ( $N$-[4-methoxy-5-[[4-(1-methylindol3-yl)pyrimidin-2-yl]amino]-2-[methyl-[2-(methylamino)ethyl] amino]phenyl]prop-2-enamide)] appear to be extensively metabolized primarily via cytochrome P450 (P450) CYP3A4 and/or CYP3A5 as the principal P450 isozymes. Other cytochromes may also contribute to the metabolism of osimertinib in terms of potential drug-drug interactions, and hence any drug-drug interaction owing to CYP3A4/5 would be moderated by the existence of alternative metabolic and elimination routes, including covalent binding to proteins, glutathione, and cysteinyl glycine (Dickinson et al., 2016). Studies have shown that neither gastric $\mathrm{pH}$ nor coadministration of osimertinib with food has a clinically significant effect on osimertinib exposure (Vishwanathan et al., 2018a). Moreover, no clinically relevant effect on osimertinib exposure was observed after coadministration of the strong CYP3A inhibitor itraconazole (Vishwanathan et al., 2016), Population PK analysis indicated that osimertinib exposure is not affected to a clinically relevant extent by patients' age, gender, body weight, ethnicity, or renal or mild hepatic impairment based on National Cancer Institute-Organ Dysfunction Working Group (NCI-ODWG) criteria (Brown et al., 2017).

Herein, we report the results of a phase 1 trial (NCT02161770) designed to characterize the impact of mild or moderate hepatic impairment (based on Child-Pugh criteria) on the PK of osimertinib and its metabolites (AZ5104 and AZ7550) in patients with advanced solid tumors. These results are also compared with a population PK study that used the NCIODWG classification for hepatic impairment. This study was designed in accordance with US Food and Drug Administration (FDA) guidance on the assessment of the influence of hepatic impairment on drug PK (https://www.fda.gov/downloads/ Drugs/GuidanceComplianceRegulatoryInformation/Guidances/ ucm072123.pdf). According to current understanding of the science of hepatic impairment, clinical studies on hepatic impairment are considered the best approach for this evaluation. For an overview and comparison of Child-Pugh and NCIODWG classification criteria, please refer to Supplemental Material.

\section{Methods}

Trial Design. This was a phase 1, open-label, nonrandomized twopart study. In part A (PK phase), eligible patients received a single $80 \mathrm{mg}$ oral dose of osimertinib on day 1 and returned for assessments on days 3,4 , $6,8,10,15$, and 22 (Supplemental Fig. S1). In part B (safety assessment), patients could continue to receive osimertinib $80 \mathrm{mg}$ once daily after completion of part A, if deemed clinically appropriate by the investigator.

The primary objective was to characterize the effect of hepatic impairment on the PK of a single oral dose of osimertinib, $80 \mathrm{mg}$ (part A). Secondary objectives included characterization of the effect of hepatic impairment on the PK of osimertinib metabolites (AZ5104 and AZ7550) after a single dose and to investigate the safety and tolerability of single-dose and continuous dosing of osimertinib in the same patient groups (parts A and B).

The study was conducted in accordance with International Conference on Harmonization-Good Clinical Practice guidance; protocols were reviewed and approved by an independent ethics committee or institutional review board in each participating country before implementation. Informed consent was obtained from all study participants. The study protocol was written in accordance with FDA and European Medicines Agency guidance on hepatic impairment and was approved by both agencies before initiation of the study. Data underlying the findings described in this article may be obtained in accordance with AstraZeneca's data sharing policy described at https:// astrazenecagrouptrials.pharmacm.com/ST/Submission/Disclosure.

Participants. Patients were aged $\geq 18$ years, Eastern Cooperative Oncology Group performance status $\leq 2$, and with histologic or cytologic confirmation of any malignant solid tumor refractory or resistant to standard therapy or for whom no suitable effective standard therapy exists. Patients with asymptomatic, stable central nervous system metastases were permitted. Previous cancer treatments had to be completed before study entry (see Supplemental Information).

For the hepatic impairment groups, patients had to have stable chronic hepatic impairment for at least 2 weeks before the start of the study that fulfilled the criteria for mild or moderate hepatic impairment as defined by the Child-Pugh criteria (Child-Pugh A and B, respectively) [Child-Pugh scoring (Table 1)] and interpretation and NCI-ODWG classification criteria (Table 2)]. Patients with hepatic metastases and/or hepatocellular carcinoma were eligible for the study, provided the metastases were not the sole reason for any changes in liver function. Patients had to have globally impaired hepatic function to participate in the study. Patients were ineligible if they had developed hepatic encephalopathy within the last 4 weeks before Day 1. Patients with previous episodes of hepatic encephalopathy who were stable on treatment of at least 4 weeks' duration before Day 1 were allowed.

Inclusion criteria for normal hepatic function included a negative result for serum hepatitis B surface antigen and hepatitis $\mathrm{C}$ antibody, total bilirubin $\leq 1.5 \times$ the institutional upper limit of normal (ULN), albumin and prothrombin time within normal limits, no ascites or encephalopathy, and aspartate aminotransferase (AST) or alanine aminotransferase $\leq 2.5 \times$ ULN unless liver metastases were present, in which case it had to be $<5 \times \mathrm{ULN}$.

Safety and Tolerability. Safety assessments included adverse event (AE) reporting graded by Common Terminology Criteria for Adverse Events (version 4.0), physical examination, ophthalmologic examination, electrocardiogram, clinical chemistry, hematology, and urinalysis.

Sample Collection and Bioanalysis. Plasma samples were collected for PK analysis at predose and at 1, 1.5, 2, 3, 4, 6, 8, 10, $12,24,48,72,120,168,216,334$, and 506 hours postdose and for protein-binding analysis at 6, 24, 48, and 168 hours. Urine samples were collected at predose and 0-24 hours. These samples were analyzed by Covance Laboratories (Harrogate, UK) using validated bioanalytical methods.

Calibration, quality control, and clinical study samples (40 $\mu \mathrm{l})$ were spiked with $\left({ }^{13} \mathrm{C},{ }^{2} \mathrm{H} 3\right)$ osimertinib as an internal standard, processed by either protein precipitation (plasma method) or dilution (urine and plasma ultrafiltrate), and then simultaneously assayed for osimertinib, AZ5104, and AZ7550 using reversed-phase high performance liquid chromatography with Turbo Ion Spray tandem mass spectrometric detection (AB Sciex, Framingham, MA, USA). Drug-to-internal standard peak area ratios for the standards were used to create either a linear or quadratic calibration curve using 1/X2 weighted least-squares regression analysis. Concentrations of each analyte were quantified by comparing the ratios for each in a trial sample with those in the relevant calibration curve. No analytically significant interferences from endogenous matrix components were observed at the retention times of each analyte in the matrix samples screened. All methods demonstrated acceptable selectivity with mean, normalized matrix factors of $1.00 \pm 0.08$ observed at the concentrations tested. The limit of quantification (LOQ) was $0.05 \mathrm{nM}$ for osimertinib and $0.0515 \mathrm{nM}$ for AZ5104 and AZ7550 in all matrices. The accuracy ranged from $95 \%$ to $108 \%$, and precision ranged from $1.7 \%$ to $10.3 \%$ for all analytes across all matrices. 
TABLE 1

Child-Pugh hepatic dysfunction classification criteria and interpretation (Pugh et al., 1973)

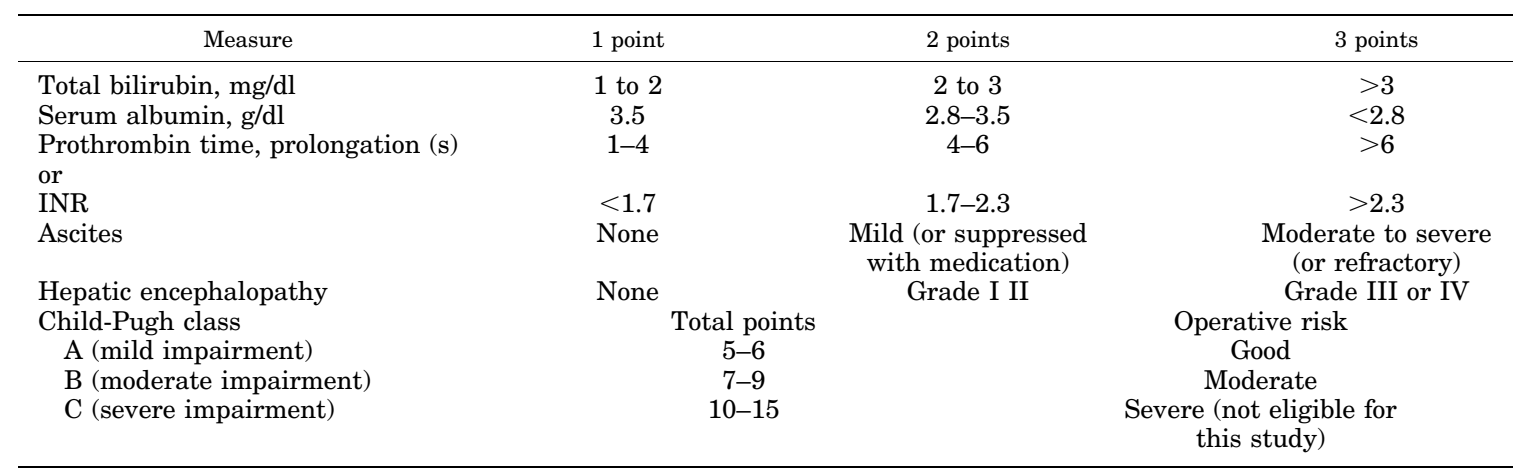

INR, international normalized ratio.

PK Analysis. The PK parameters were derived using noncompartmental methods with Phoenix WinNonlin Version 6.4 (Pharsight Corp., A Certara Company, Princeton, NJ) and/or SAS version 9.2 (SAS Institute, Inc., Cary, NC). The actual elapsed time from dose was used for plasma PK parameter calculations. All descriptive and inferential statistical computations were performed using SAS version 9.2 statistical summaries, and analyses of non-PK data were performed by QuintilesIMS using SAS version 9.2. PK sample bioanalysis was performed by Covance Laboratories.

Statistical Methods. The sample size was based on FDA hepatic guidance, which recommends inclusion of at least six patients in each subgroup (normal, mild, and moderate hepatic impairment) (https://www. fda.gov/downloads/Drugs/GuidanceComplianceRegulatoryInformation/ Guidances/ucm072123.pdf). To account for a withdrawal rate of approximately $20 \%$, we planned to enroll 26 patients (10 normal, 8 mild, and 8 moderate) to obtain 20 evaluable (eight normal, six mild, and six moderate) patients.

The PK analysis set was defined as patients with PK sampling data collected postdose without any important deviations or events that could alter data evaluation. The safety analysis set was all patients who received at least one dose of osimertinib and had postdose data available.

We summarized the PK parameters for plasma osimertinib, AZ5104, and AZ7550 using the appropriate descriptive statistics. Natural log-transformed area under the plasma concentration-time curve (AUC), AUC from zero to last quantifiable concentration at time $t$ $\left(\mathrm{AUC}_{0-\mathrm{t}}\right.$ ), and $C_{\max }$ were compared between hepatic function groups using analysis of variance, with hepatic function group (normal, mild, and moderate) as a fixed effect. Comparisons between mild hepatic impairment and moderate hepatic impairment versus normal hepatic function were performed. The mean differences and corresponding confidence intervals (CIs) were back-transformed to the original scale to give estimates of the ratios (mild vs. normal and moderate vs. normal) and the associated $90 \%$ CIs. Additionally, backtransformed geometric means, together with 95\% CIs for AUC (and/or $\mathrm{AUC}_{0-\mathrm{t}}$ ) and $C_{\text {max }}$, were estimated for each hepatic function group.

For osimertinib and its metabolites, the relationships between hepatic functional variables (albumin and total bilirubin collected on
Day -1 ) and AUC and $C_{\max }$ were assessed using a regression model, with hepatic function value included as the independent variable, and PK parameter as the dependent variable. Slopes, intercepts, and associated $90 \%$ CIs were presented along with the coefficient of determination.

Population PK Analysis and NCI-ODWG Hepatic Impairment Criteria. An additional comprehensive assessment of the impact of hepatic impairment on the PK of osimertinib in patients with NSCLC was undertaken using a population PK analysis, using NCI-ODWG as the hepatic impairment criteria. Previously published population PK analysis methods (Brown et al., 2017) updated with additional clinical data were used in this analysis. The population PK data set included patient data from phase 1, 2, and 3 osimertinib trials AURA (NCT01802632), AURA2 (NCT02094261), AURA3 (NCT02151981), and FLAURA (NCT02296125) (Jänne et al., 2015; Goss et al., 2016; Mok et al., 2017; Yang et al., 2017; Soria et al., 2018). Details of the studies included in the population PK dataset are provided in Supplemental Table S1. Osimertinib tablet formulation was used in all pivotal and drug-drug interaction studies.

\section{Results}

Patients. Overall, 49 patients were screened, and 30 were assigned to treatment. Ten patients were included in the normal hepatic function group, seven in the mild hepatic impairment group, and five in the moderate hepatic impairment group. Eight patients were determined, after receiving treatment in part A, to have been incorrectly enrolled in the mild hepatic impairment group because they did not have stable global hepatic impairment and were excluded from the PK analysis (these patients fit into Child-Pugh mild criteria based on their liver function, ascites, and encephalopathy, but it was found to be acute rather than chronic liver impairment). These patients are included in the group listed as "other"; details on their exclusion are shown in the Supplemental Information. The PK analysis set included the 22 patients who

TABLE 2

NCI-ODWG hepatic dysfunction classification criteria

\begin{tabular}{lccccc}
\hline Group liver function & Group A Normal & Group B Mild & Group C Moderate & Group D Severe & Group E Liver Transplant \\
\hline Total bilirubin & $\leq \mathrm{ULN}$ & B1: $\leq \mathrm{ULN}$ & $>1.5 \times-3 \times$ ULN & $>3 \times$ ULN & Any \\
SGOT/AST & $\leq \mathrm{ULN}$ & B2: $>1.0 \times-1.5 \times$ ULN & Any & Any & Any \\
& & B2: Any & & & \\
\hline
\end{tabular}

AST, aspartate transaminase; INR, International Normalized Ratio; NCI-ODWG, National Cancer Institute-Organ Dysfunction Working Group; SGOT, serum glutamic oxaloacetic transaminase; ULN, upper limit of normal. 
were correctly enrolled; the safety analysis set for part A included all 30 patients; part B included 24 patients. Six patients did not enter part $B$ for the following reasons: three died, two discontinued because of worsening disease, and one discontinued owing to an $\mathrm{AE}$ (thrombocytopenia, which started pretreatment on Day -1).

Baseline demographics and disease characteristics are summarized in Table 3. Median ages of the mild and moderate hepatic impairment groups (70.0 and 57.0 years, respectively) were within 10 years of the normal patient group (62.0 years). There were more male patients in the hepatic impairment groups than the normal group. Mean body mass index in the mild and moderate hepatic impairment groups $\left(26.5 \mathrm{~kg} / \mathrm{m}^{2}\right.$ and $23.6 \mathrm{~kg} / \mathrm{m}^{2}$, respectively) was within $15 \%$ of the normal hepatic function group $\left(25.0 \mathrm{~kg} / \mathrm{m}^{2}\right)$. The most common primary tumor location was the liver (six of seven patients in the mild hepatic impairment group and two of five in the moderate hepatic impairment group).

Osimertinib PK. Geometric mean osimertinib plasma concentrations were lower in patients with mild or moderate hepatic impairment compared with patients with normal hepatic function over time; however, these levels were similar in patients with mild and moderate hepatic impairment (Fig. 1). PK parameters showed a lower AUC and $C_{\max }$ for osimertinib in patients with mild or moderate hepatic impairment relative to those with normal hepatic function (Table 4). In patients with mild hepatic impairment, osimertinib exposure, based on $C_{\max }$ and AUC, was $51.43 \%$ and $63.25 \%$, respectively, relative to patients with normal hepatic function. Similarly, in patients with moderate hepatic impairment, osimertinib exposure was $60.67 \%$ and $68.36 \%$, respectively (Table 4 ).

Time of maximum concentration $\left(t_{\max }\right)$ was slightly shorter in patients with hepatic impairment, and terminal half-life $\left(t_{1 / 2 \lambda z}\right)$ was slightly longer in the mild hepatic impairment group compared with patients with normal hepatic function; $t_{1 / 2 \lambda z}$ was similar in patients with normal and moderate hepatic impairment. Apparent plasma clearance $(\mathrm{CL} / \mathrm{F})$ and apparent volume of distribution $(\mathrm{Vz} / \mathrm{F})$ were greater in patients with hepatic impairment than in normal patients. Renal clearance was low in all groups.

Albumin levels accounted for less than $1 \%$ of the interpatient variability in osimertinib exposure, and the slopes of the linear regressions were not different from zero $\left(C_{\max } P=0.8076\right.$, AUC $P=0.6874)$. Total bilirubin levels accounted for approximately $20 \%$ of the interpatient variability in osimertinib exposure, and the negative slopes of the linear regressions were significantly different from zero $\left(C_{\max } P=0.0361\right.$, AUC $P=0.0484$ ); thus, higher values of total bilirubin were accompanied with lower osimertinib exposure (Supplemental

TABLE 3

Baseline demographics and disease characteristics

ECOG performance status and overall disease classification are based on assessments at baseline.

\begin{tabular}{|c|c|c|c|c|c|}
\hline & \multicolumn{5}{|c|}{ No. (\%) of patients } \\
\hline & $\begin{array}{l}\text { Normal Hepatic Function } \\
\qquad(n=10)\end{array}$ & $\begin{array}{l}\text { Mild Hepatic Impairment } \\
(n=7)\end{array}$ & $\begin{array}{l}\text { Moderate Hepatic } \\
\text { Impairment }(n=5)\end{array}$ & $\begin{array}{l}\text { Other }^{a} \\
(n=8)\end{array}$ & $\begin{array}{l}\text { Total } \\
(n=30)\end{array}$ \\
\hline Age (y), median (range) & $62.0(56,73)$ & $70.0(57,79)$ & $57.0(52,67)$ & $64.5(51,78)$ & $61.0(51,79)$ \\
\hline \multicolumn{6}{|l|}{ Sex, $n(\%)$} \\
\hline Male & $4(40)$ & $6(86)$ & $5(100)$ & $2(25)$ & $17(57)$ \\
\hline Female & $6(60)$ & $1(14)$ & 0 & $6(75)$ & $13(43)$ \\
\hline \multicolumn{6}{|l|}{ Race, $n(\%)$} \\
\hline White & $9(90)$ & $7(100)$ & $4(80)$ & $8(100)$ & $28(93)$ \\
\hline Black or African American & $1(10)$ & 0 & 0 & 0 & $1(3)$ \\
\hline Asian & 0 & 0 & $1(20)$ & 0 & $1(3)$ \\
\hline \multicolumn{6}{|l|}{ Smoking status, $n(\%)$} \\
\hline Former & $3(30)$ & $5(71)$ & $4(80)$ & $2(25)$ & $14(47)$ \\
\hline Never & $6(60)$ & $1(14)$ & $1(20)$ & $4(50)$ & $12(40)$ \\
\hline Current & $1(10)$ & $1(14)$ & 0 & $2(25)$ & $4(13)$ \\
\hline \multicolumn{6}{|l|}{ ECOG PS, $n(\%)$} \\
\hline 0 & $4(40)$ & $5(71)$ & $1(20)$ & $2(25)$ & $12(40)$ \\
\hline 1 & $6(60)$ & $2(29)$ & $4(80)$ & $6(75)$ & $18(60)$ \\
\hline \multicolumn{6}{|l|}{ Primary tumor location, $n(\%)$} \\
\hline Liver & 0 & $6(86)$ & $2(40)$ & $1(13)$ & $9(30)$ \\
\hline Lung & $3(30)$ & 0 & $1(20)$ & $1(13)$ & $6(20)$ \\
\hline Cervix & $1(10)$ & 0 & 0 & $1(13)$ & $2(7)$ \\
\hline $\begin{array}{l}\text { Head and neck (including } \\
\text { nasopharynx, larynx, trachea) }\end{array}$ & $1(10)$ & 0 & $1(20)$ & 0 & $2(7)$ \\
\hline Prostate & $1(10)$ & 0 & 0 & $1(13)$ & $2(7)$ \\
\hline Other & $1(10)$ & 0 & 0 & $1(13)$ & $1(3)$ \\
\hline Breast & 0 & 0 & 0 & $1(13)$ & $1(3)$ \\
\hline Buccal & 0 & $1(14)$ & 0 & 0 & $1(3)$ \\
\hline Colon & 0 & 0 & $1(20)$ & 0 & $1(3)$ \\
\hline Ovary & $1(10)$ & 0 & 0 & 0 & $1(3)$ \\
\hline Pancreas & 0 & 0 & 0 & $1(13)$ & $1(3)$ \\
\hline Rectal & $1(10)$ & 0 & 0 & 0 & $1(3)$ \\
\hline Renal & $1(10)$ & 0 & 0 & 0 & $1(3)$ \\
\hline \multicolumn{6}{|l|}{ Overall disease classification } \\
\hline Metastatic $^{b}$ & $7(70)$ & $4(57)$ & $5(100)$ & $7(88)$ & $23(77)$ \\
\hline Locally advanced ${ }^{c}$ & $3(30)$ & $3(43)$ & 0 & $1(13)$ & $7(23)$ \\
\hline
\end{tabular}

ECOG, Eastern Cooperative Oncology Group; PS, performance status.

${ }^{a}$ Patients not meeting inclusion criteria for a protocol-defined hepatic impairment group.

${ }^{b}$ Metastatic disease: patient has any metastatic site of disease.

${ }^{c}$ Locally advanced: patient has only locally advanced sites of disease. 


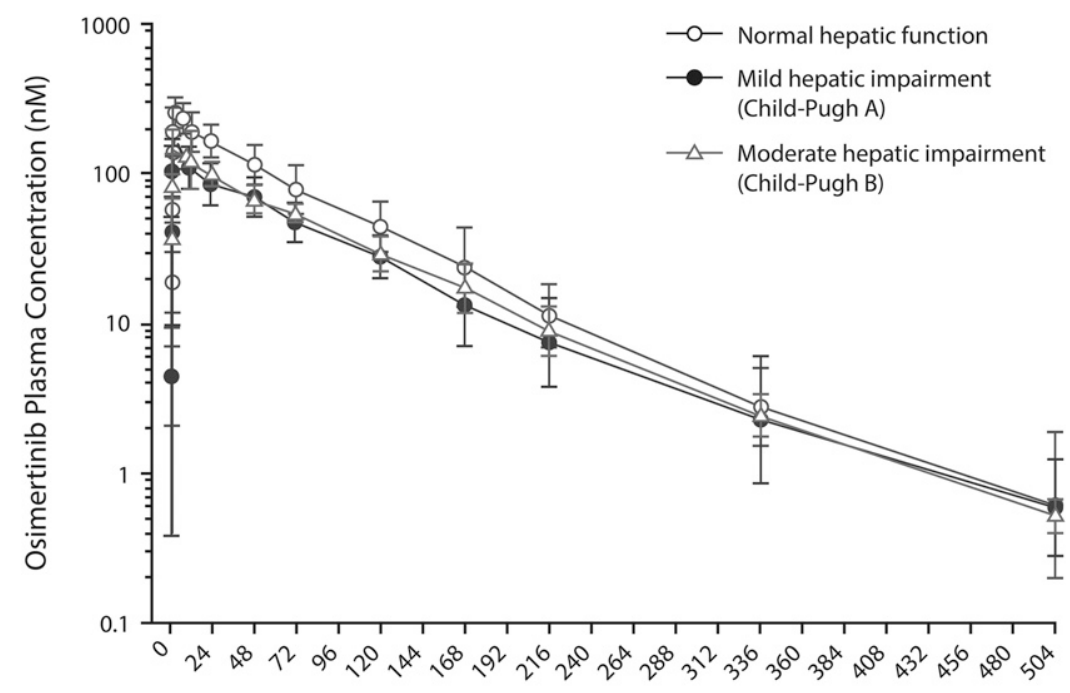

Fig. 1. Geometric mean plasma concentration of osimertinib by hepatic function group (semi-logarithmic scale; pharmacokinetics analysis set). Geometric mean S.D. expressed in the error bars as the exponential of (mean of the log concentration \pm S.D. of the log concentration)

Time (hours)

Table S2). Inclusion of the baseline bilirubin from this study with other studies from the osimertinib clinical program showed no relationship observed between baseline bilirubin and osimertinib exposure (Fig. 2).

The protein-free fraction of osimertinib was above the LOQ $(0.025 \mathrm{nM})$ in only one patient (mild impairment patient with $0.095 \mathrm{nM}$ at 6 hours, which represented $0.04 \%$ of the total concentration at that time point), whereas it was lower than the LOQ in all other patients in all cohorts at all time points. Therefore, it was not possible to determine the changes in free fraction levels of osimertinib owing to hepatic impairment.

Osimertinib Metabolite PK Parameters. Exposure to the metabolites of osimertinib was approximately $30 \%-50 \%$ lower in patients with mild and moderate hepatic impairment relative to patients with normal hepatic function (Table 4); however, AZ5104 and AZ7750 metabolites to parent ratios for $C_{\text {max }}$ and AUC were similar in patients with mild or moderate hepatic impairment and in patients with normal hepatic function; overall, this amounted to less than $10 \%$ of osimertinib exposure. No relationship was found between albumin levels and osimertinib or metabolite exposure. Bilirubin accounted for approximately $30 \%$ of the interpatient variability in AZ5104 exposure, where an increase in bilirubin was accompanied by a decrease in exposure. We found no relationship between bilirubin and AZ7550 exposure.

Comparison of Hepatic Impairment Classification Criteria. The population PK model-derived osimertinib AUC was plotted as a function of hepatic dysfunction, as defined by NCI-ODWG criteria, and then compared with a plot of osimertinib AUC as a function of hepatic impairment as defined by the Child-Pugh criteria using data from this study (Fig. 3). The medians and interquartile ranges for osimertinib AUC overlapped considerably between patients with normal hepatic function as classified by NCI-ODWG and the ChildPugh criteria, as did those for mild and moderate hepatic impairment. Based on the population PK analysis of patients with NSCLC and treated with osimertinib, where the NCIODWG criteria for hepatic dysfunction were applied, mild or moderate hepatic impairment had no impact on the $\mathrm{CL} / \mathrm{F}$ of osimertinib. Population PK data set values for PK parameters are shown in Table 4.
Safety. In part B, the mean total treatment duration was 3.9 months (S.D., 5.6), with a median of 1.9 months (range, 0.3-24.0). The mean actual treatment duration was 3.8 months (S.D., 5.5), with a median of 1.9 months (range, 0.3-24.0). Mean total treatment and mean actual treatment durations were longer in patients with normal hepatic function than in patients with mild or moderate hepatic impairment or in the "other" group.

In part $\mathrm{A}$, grade $\geq 3, \mathrm{AEs}$ were reported in six patients $(20 \%)$, none of whom were considered by the investigator to be possibly related to treatment with osimertinib. In part B, grade $\geq 3$ AEs were reported in eight patients (33\%); two were considered possibly related to osimertinib (vomiting and stomatitis) (Supplemental Table S3). AEs of special interest reported in parts A and B are summarized in Supplemental Table S3. Serious adverse events (SAEs) were reported in three patients (10\%) in part A and in six patients $(25 \%)$ in part B (see Supplemental Information for details on SAEs). The investigator did not consider any of the SAEs to be possibly related to osimertinib.

No AEs of interstitial lung disease were reported in either part of the study. AEs leading to death occurred in two patients (acute kidney injury and meningitis/pneumonia aspiration), both of which were unrelated to study treatment.

\section{Discussion}

This study was designed in accordance with FDA guidance (https://www.fda.gov/downloads/Drugs/GuidanceComplianceRegulatoryInformation/Guidances/ucm072123.pdf) to assess the impact of mild and moderate stable global hepatic impairment (using the Child-Pugh classification) on the PK profile of osimertinib in patients with advanced solid tumors after a single $80 \mathrm{mg}$ dose of osimertinib. In line with FDA guidance (https://www.fda.gov/downloads/Drugs/GuidanceComplianceRegulatoryInformation/Guidances/ucm072123.pdf), a single-dose PK analysis was considered appropriate and representative of steady-state PK as previous investigations have shown linear PK for osimertinib, such that single-dose osimertinib exposure is predictive of multiple dosing, indicating there is 
TABLE 4

Pharmacokinetic parameters of osimertinib, AZ5104, and AZ7550 for each hepatic function group (pharmacokinetic analysis set)

Comparisons are based on an analysis of variance model with a fixed effect for hepatic function group.

\begin{tabular}{|c|c|c|c|c|}
\hline Pharmacokinetic Parameter & $\begin{array}{l}\text { Normal Hepatic } \\
\text { Function }^{a}(n=10)\end{array}$ & $\begin{array}{c}\text { Mild Hepatic } \\
\text { Impairment }(n=7)\end{array}$ & $\begin{array}{l}\text { Moderate Hepatic } \\
\text { Impairment }(n=5)\end{array}$ & $\begin{array}{c}\text { Population PK (Based on } \\
n=1367 \text { Patients) }\end{array}$ \\
\hline \multicolumn{5}{|l|}{ Osimertinib } \\
\hline AUC (nM·h), geometric mean (\%GCV) & $15,780(38)$ & $9983(36)$ & $10,790(22)$ & $11,040^{b}$ \\
\hline $\begin{array}{l}\text { Comparison: mild }(n=7) \text { vs. normal } \\
(n=9) ; \text { ratio }(90 \% \mathrm{CI})\end{array}$ & & $63.25(47.34,84.51)$ & & \\
\hline $\begin{array}{l}\text { Comparison: moderate }(n=5) \mathrm{vs} \text {. } \\
\text { normal }(n=9) ; \text { ratio }(90 \% \mathrm{CI})\end{array}$ & & $68.36(49.60,94.21)$ & & \\
\hline$C_{\max }(\mathrm{nM})$, geometric mean $(\% \mathrm{GCV})$ & $291.8(45)$ & $150.1(37)$ & $177.1(41)$ & $509^{b}$ \\
\hline $\begin{array}{l}\text { Comparison: mild }(n=7) \text { vs. normal } \\
\quad(n=10) ; \text { ratio }(90 \% \mathrm{CI})\end{array}$ & & $51.43(36.58,72.31)$ & & \\
\hline $\begin{array}{l}\text { Comparison: moderate }(n=5) \text { vs. } \\
\text { normal }(n=10) ; \text { ratio }(90 \% \text { CI })\end{array}$ & & $60.67(41.55,88.60)$ & & \\
\hline $\mathrm{t}_{\max }(\mathrm{h})$, median $(\min , \max )$ & $4.00(1.50,11.83)$ & $3.00(1.98,8.00)$ & $3.00(1.50,6.00)$ & NA \\
\hline$t_{1 / 2 \lambda z}(\mathrm{~h})$, mean (S.D.) & $69.05(10.46)$ & $82.84(19.33)$ & $71.88(6.581)$ & $44(3.0)^{c}$ \\
\hline CL/F (liters/h), mean (S.D.) & $10.76(3.914)$ & $16.85(5.538)$ & $15.12(3.224)$ & $14.3(1.4)^{d}$ \\
\hline Vz/F (liters), mean (S.D.) & $1038(351.7)$ & $1937(570.1)$ & $1560(318.5)$ & $918(3.3)^{d}$ \\
\hline $\mathrm{CL}_{\mathrm{R}}$ (liters/h), mean (S.D.) & $0.07879(0.06086)$ & $0.1651(0.09735)$ & $0.04419(0.04575)$ & - \\
\hline \multicolumn{5}{|l|}{ AZ5104 } \\
\hline AUC (nM·h), geometric mean (\%GCV) & $1387(60)$ & $921.8(47)$ & $705.2(43)$ & - \\
\hline $\mathrm{C}_{\max }(\mathrm{nM})$, geometric mean (\%GCV) & $9.793(47)$ & $6.489(44)$ & $4.312(50)$ & - \\
\hline$t_{\max }(\mathrm{h})$, median $(\min , \max )$ & $12.05(4.00,48.83)$ & $24.10(3.00,48.02)$ & $48.22(12.00,72.00)$ & - \\
\hline$t_{1 / 2 \lambda z}(\mathrm{~h})$, mean (S.D.) & $72.96(10.72)$ & $82.34(21.28)$ & $78.15(10.88)$ & - \\
\hline MRC $_{\max }$, mean (S.D.) & $0.03586(0.01386)$ & $0.04707(0.02183)$ & $0.02687(0.01481)$ & - \\
\hline MRAUC, mean (S.D.) & $0.09184(0.02926)$ & $0.09869(0.04466)$ & $0.06873(0.02476)$ & - \\
\hline $\mathrm{CL}_{\mathrm{R}}(\mathrm{l} / \mathrm{h})$, mean (S.D.) & $0.2859(0.1632)$ & $0.9207(0.4934)$ & $0.2983(0.2240)$ & - \\
\hline \multicolumn{5}{|l|}{ AZ7550 } \\
\hline AUC (nM·h), geometric mean (\%GCV) & $879.8(41)$ & $588.7(35)$ & $579.6(37)$ & - \\
\hline$C_{\max }(\mathrm{nM})$, geometric mean $(\% \mathrm{GCV})$ & $4.929(31)$ & $3.232(42)$ & $3.005(73)$ & - \\
\hline$t_{\max }(\mathrm{h})$, median $(\min , \max )$ & $9.95(6.00,72.02)$ & $12.00(6.00,47.58)$ & $23.92(1.50,48.22)$ & - \\
\hline$t_{1 / 2 \lambda z}(\mathrm{~h})$, mean (S.D.) & $104.0(21.84)$ & $104.2(16.99)$ & $98.66(11.11)$ & - \\
\hline $\mathrm{MRC}_{\max }$, mean (S.D.) & $0.01810(0.006706)$ & $0.02508(0.01502)$ & $0.01859(0.008441)$ & - \\
\hline MRAUC, mean (S.D.) & $0.05686(0.01200)$ & $0.06694(0.03260)$ & $0.05739(0.02283)$ & - \\
\hline $\mathrm{CL}_{\mathrm{R}}(\mathrm{l} / \mathrm{h})$, mean (S.D.) & $0.6092(0.4695)$ & $1.247(0.3285)$ & $0.3681(0.1514)$ & - \\
\hline
\end{tabular}

AUC, area under the plasma-concentration time curve from zero to infinity; CL/F, apparent plasma clearance; $\mathrm{CL}_{\mathrm{R}}$, renal clearance; $C_{\mathrm{max}}$, maximum plasma concentration;

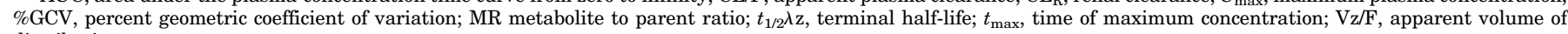
distribution.

${ }^{a}$ Normal = normal hepatic function; Mild = mild hepatic impairment [Child-Pugh A]; moderate = moderate hepatic impairment [Child-Pugh B].

${ }^{b}$ AUCss and $\mathrm{Css}_{\max }$ at steady state shown.

${ }^{c} t_{1 / 2}$ shown.

${ }^{d}$ Population typical value (\% relative S.E.).

no change in osimertinib metabolism over time (Planchard et al., 2016; Brown et al., 2017).

Logically, if hepatic impairment affected the elimination of osimertinib, it would result in an increase in osimertinib exposure compared with patients with normal hepatic function; however, in the population evaluated in this study, exposure to osimertinib and its metabolites was approximately $30 \%-50 \%$ lower in patients with mild and moderate hepatic impairment than in patients with normal hepatic function. It is possible that the lower exposure observed in patients with mild and moderate hepatic impairment may be due to decreased absorption or reduced bioavailability, as witnessed by the decrease in $C_{\max }$ relative to patients with normal hepatic function; however, the biologic reason for this lower absorption owing to hepatic impairment is unclear, and to our knowledge, there is no scientific evidence or literature precedence to demonstrate lower absorption or reduced bioavailability owing to hepatic impairment. The absolute bioavailability of osimertinib is $70 \%$ with minimal first-pass metabolism (Vishwanathan et al., 2019). The mean exposure to osimertinib and its metabolites in patients with normal hepatic function in this study was greater than has been observed in previous studies (mean AUC of 15,000 nM.h in this study compared with $10,000-11,000 \mathrm{nM} \cdot \mathrm{h}$ in other studies) and greater than the typical values expected in patients with normal hepatic function (population PK mean area under the plasma concentration time curve at steady state (AUCss) of 11,040 nM-h) (Planchard et al., 2016; Brown et al., 2017). Therefore, the reduced exposure in patients with mild to moderate hepatic impairment relative to patients with normal hepatic impairment may be exaggerated because of the greater mean exposure observed in this small number of normal hepatic function cohort. Moreover, in the population $\mathrm{PK}$ analysis, mean exposure in the overall population $(11,040 \mathrm{nM} \cdot \mathrm{h})$ was similar to that in patients with mild and moderate hepatic impairment (AUC: 9983 and $10,790 \mathrm{nM} \cdot \mathrm{h}$, respectively). Furthermore, based on the absorption rate constant of $0.196 / \mathrm{h}$, the $\mathrm{CL} / \mathrm{F}$ of $14.3 \mathrm{liter} / \mathrm{h}$ and volume of distribution of 918 liters, the predicted single dose mean $C_{\text {max }}$ would be $128 \mathrm{nM}$ (90\% prediction interval: $27.7-449$ ), which is in a range similar to the values observed in this study. The single oral dose geometric mean (range) $C_{\max }$ of osimertinib at $80 \mathrm{mg}$ in the food-effect study was $218 \mathrm{nM}(95.2-381)$ (Vishwanathan et al., 2018a) and in the CYP3A4 interaction study was $242.5 \mathrm{nM}$ (88.8-704) (Vishwanathan et al., 2018b). The higher level of exposure than expected in the normal hepatic function group possibly reflects the limited number of patients evaluated in this study who were still within the range of exposures. This is an inherent limitation of trials studying $\mathrm{PK}$ in small numbers of patients with hepatic dysfunction. 


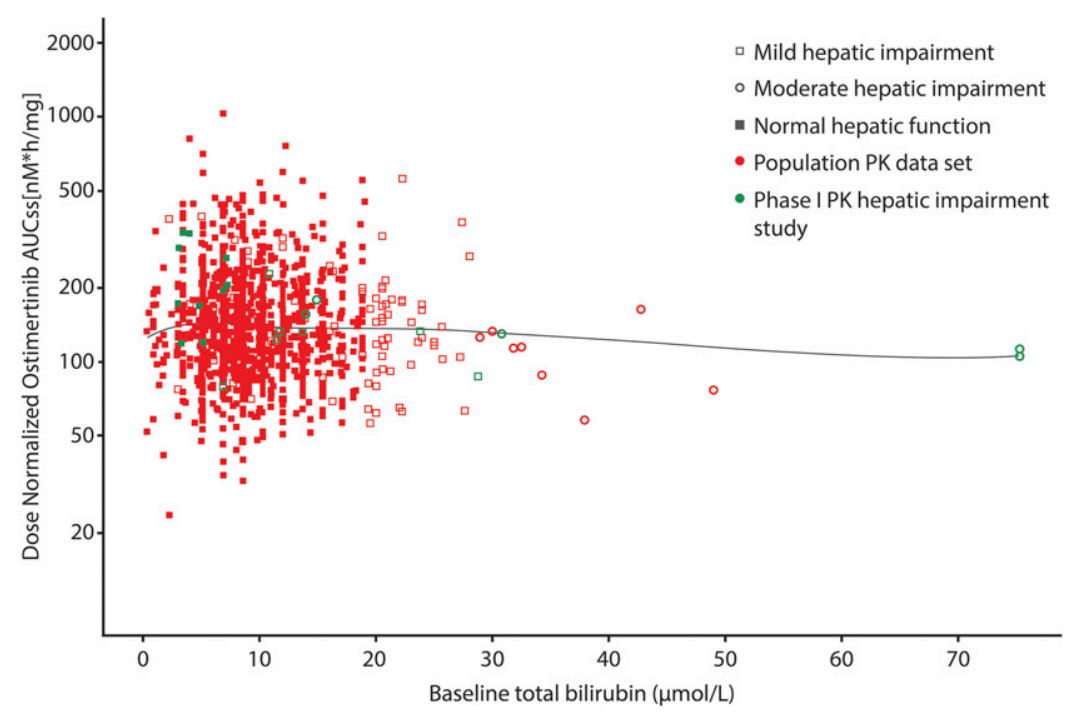

Fig. 2. Individual osimertinib AUCss as a function of baseline bilirubin level in patients. Circles represent individual AUCss values based on population PK analysis. AUCss area under the plasma concentration time curve at steady state; LOESS, local polynomial regression.
Another factor that could have contributed to the difference in exposure in hepatic impairment patients compared with normal subjects is plasma protein binding (Roberts et al., 2013). Osimertinib is an acidic drug that binds to albumin, and in many advanced cancer patients (as well as in patients with hepatic impairment), hypoalbuminemia is very common; therefore, altered protein binding may occur. In this study, however, the extent and clinical consequences of this are not known since the free exposure could not be determined.

No relationship was found between albumin levels and osimertinib or metabolite exposure. Bilirubin is a breakdown product of hemolysis, which is normally removed from the blood by liver cells to be excreted in bile; therefore, elevated bilirubin levels are an indication of hepatic impairment (Sticova and Jirsa, 2013). Bilirubin accounted for approximately $20 \%$ and $30 \%$ of the between-patient variability in osimertinib and AZ5104, respectively. An increase in bilirubin was accompanied by a decrease in osimertinib exposure, which is the reverse of that expected; however, this relationship is likely driven by two patients who had extremely high bilirubin levels (visible at the far right of Fig. 2). In a population PK analysis of $>1300$ patients that included these two patients, bilirubin level had no overall effect on the exposure of osimertinib or AZ5104.

$\mathrm{Vz} / \mathrm{F}$ was high across all cohorts and significantly greater than the total body water volume. It was highest in patients with mild hepatic impairment (1937 liters) compared with those in the moderate hepatic impairment (1560 liters) and normal hepatic function groups (1038 liters). No clear trend owing to hepatic impairment was found, and the changes noted at such a high volume of distribution are unlikely to be clinically relevant.
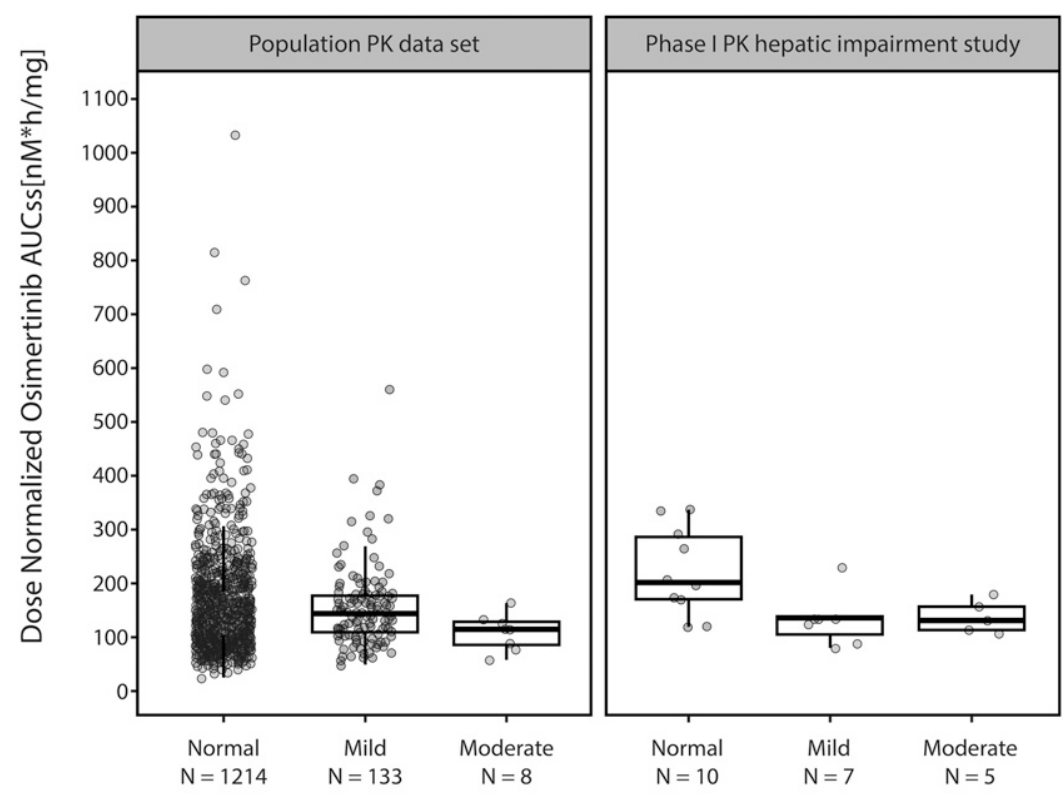

Fig. 3. Osimertinib AUC as a function of hepatic dysfunction as defined by NCI-ODWG or Child-Pugh criteria. Circles represent individual AUCss values based on population PK analysis using the NCI-ODWG criteria in the population PK set. For the phase $1 \mathrm{PK}$ hepatic impairment study, individual AUC values are shown. AUCss, area under the plasma concentration time curve at steady state; NCI-ODWG, National Cancer Institute Organ Dysfunction Working Group; PK, pharmacokinetics.

Hepatic Impairment Status 
No apparent differences in safety were noted between patients with normal hepatic function or patients with mild or moderate hepatic impairment. The safety profile observed in this study was consistent with expectations for this population and the current known safety profile for osimertinib.

A phase 1 study in healthy adult men found that the hepatic route was the major elimination pathway for osimertinib, with only $1.7 \%$ of the dose being excreted as unchanged osimertinib renally (Dickinson et al., 2016). This finding suggests that hepatic impairment could affect the elimination and thereby increase a patient's exposure to osimertinib; however, individual hepatic function markers [alanine aminotransferase, AST, and bilirubin] were shown to have no effect on osimertinib PK (Brown et al., 2017).

The NCI-ODWG criteria for hepatic dysfunction uses two objective and readily measurable parameters, total bilirubin and AST, to classify hepatic impairment and is shown to correlate with Child-Pugh (Patel et al., 2004); however, additional information regarding a patient's hepatic function would be missed (Mansfield et al., 2016). Although ChildPugh is considered the "gold standard," NCI-ODWG classification is now commonly used in oncology to evaluate hepatic impairment. In this study, both approaches provided a similar conclusion. Therefore, using the NCI criteria may be an appropriate approach for evaluation of hepatic impairment in situations where the recruitment of patients with hepatic impairment is a significant challenge. Using a population-based approach for such evaluations saves money, time, and unnecessary clinical study.

This study was not designed to assess osimertinib PK in patients with severe hepatic impairment. Identification and recruitment of patients with stable but severe hepatic impairment (based on Child-Pugh criteria) and advanced cancer, with a life expectancy appropriate for enrollment into a clinical study, are challenging. Furthermore, the number of patients with EGFRm-positive tumors and severe hepatic impairment who are eligible for inclusion is likely to be very low. Osimertinib has an absolute bioavailability of 70\%, with multiple mechanisms contributing to its elimination (Dickinson et al., 2016), so the potential for significant increase in its exposure from hepatic impairment is likely to be low. As noted in the drug-drug interaction study with strong CYP3A4 inhibitor, itraconozale, osimertinib exposure was not increased to a clinically significant extent (24\% increase in AUC) (Vishwanathan et al., 2018b).

This study was performed in cancer patients because of dosing restrictions in healthy volunteers. Although PK can differ between healthy volunteers and cancer patients, there is no literature of drug PK being influenced by the type of cancer. Accordingly, this study was conducted in relapsed or refractory cancer patients. This was deemed ethical as, although osimertinib is indicated for patients with EGFR-mutated NSCLC, the patient population for this study included patients resistant to standard therapy or those for whom no suitable effective standard therapy exists; however, this does highlight a limitation of this study. It is plausible that the PK of osimertinib, which binds irreversibly to its target, may differ between patients with the drug target and patients without. It should be noted that population PK analysis has shown that line of therapy has no impact on the exposure of osimertinib (https://www.accessdata.fda.gov/drugsatfda_docs/ label/2018/208065s008lbl.pdf).
This study demonstrates that PK exposure of osimertinib is not increased in patients with mild or moderate hepatic impairment compared with that of patients with normal hepatic function. Because of the small sample size evaluated in phase 1 clinical interaction studies, a cumulative analysis using NCI-ODWG criteria could be an alternative approach to evaluate the impact of hepatic dysfunction on the PK of compounds. Based on a comprehensive population PK analysis and the clinical study results from a dedicated hepatic impairment study, dose modifications are not necessary for patients with mild or moderate hepatic impairment. Osimertinib has a broad therapeutic window, and changes in exposure within 2-fold of its typical exposure range with an $80 \mathrm{mg}$ dose does not require any dose modifications. Osimertinib treatment had an acceptable tolerability profile in patients with normal hepatic function and mild or moderate hepatic impairment, and there were no apparent differences in safety between the different groups.

\section{Acknowledgments}

We thank all the patients and their families and Natalie Griffiths of iMed Comms, an Ashfield Company, part of UDG Healthcare plc, for medical writing support that was funded by AstraZeneca in accordance with Good Publication Practice (GPP3) guidelines (http://www. ismpp.org/gpp3).

\section{Authorship Contributions}

Participated in research design: Grande, Harvey, Batlle, Ramalingam, So, Vishwanathan.

Conducted experiments: Grande, You, Batlle, Sarantopoulos, Ramalingam, Vishwanathan.

Contributed new reagents or analytic tools: Batlle, Vishwanathan. Performed data analysis: Harvey, You, Batlle, Galbraith, Sarantopoulos, Ramalingam, Mann, So, Johnson, Vishwanathan.

Wrote or contributed to the writing of the manuscript: Grande, Harvey, You, Batlle, Galbraith, Sarantopoulos, Ramalingam, Mann, So, Johnson, Vishwanathan.

\section{References}

Brown K, Comisar C, Witjes H, Maringwa J, de Greef R, Vishwanathan K, Cantarini M, and Cox E (2017) Population pharmacokinetics and exposure-response of osimertinib in patients with non-small cell lung cancer. $\mathrm{Br}$ J Clin Pharmacol 83:1216-1226.

Cross DA, Ashton SE, Ghiorghiu S, Eberlein C, Nebhan CA, Spitzler PJ, Orme JP, Finlay MR, Ward RA, Mellor MJ, et al. (2014) AZD9291, an irreversible EGFR TKI, overcomes T790M-mediated resistance to EGFR inhibitors in lung cancer. Cancer Discov 4:1046-1061.

Dickinson PA, Cantarini MV, Collier J, Frewer P, Martin S, Pickup K, and Ballard P (2016) Metabolic disposition of osimertinib in rats, dogs, and humans: insights into a drug designed to bind covalently to a cysteine residue of epidermal growth factor receptor. Drug Metab Dispos 44:1201-1212.

Goss G, Tsai CM, Shepherd FA, Ahn M-J, Bazhenova L, Crinò L, de Marinis F, Felip E, Morabito A, Hodge R, et al. (2018) CNS response to osimertinib in patients with T790M-positive advanced NSCLC: pooled data from two phase II trials. Ann Oncol 29:687-693.

Goss G, Tsai C-M, Shepherd FA, Bazhenova L, Lee JS, Chang G-C, Crino L, Satouchi M, Chu Q, Hida T, et al. (2016) Osimertinib for pretreated EGFR Thr790Metpositive advanced non-small-cell lung cancer (AURA2): a multicentre, open-label, single-arm, phase 2 study. Lancet Oncol 17:1643-1652.

Jänne PA, Ahn M-J, Kim D-W, Kim S-W, Planchard D, Ramalingam SS, Frewer P, Cantarini M, Ghiorghiu S, and Yang JC-H (2015) Phase I study of AZD9291 in patients with EGFR-TKI-resistant advanced NSCLC - updated progression free survival and duration of response data (Abstract). Ann Oncol 26:i60.

Mansfield AS, Rudek MA, Vulih D, Smith GL, Harris PJ, and Ivy SP; NCI Organ Dysfunction Working Group (2016) The effect of hepatic impairment on outcomes in phase I clinical trials in cancer subjects. Clin Cancer Res 22:5472-5479.

Mok TS, Wu Y-L, Ahn M-J, Garassino MC, Kim HR, Ramalingam SS, Shepherd FA, He Y, Akamatsu H, Theelen WSME, et al.; AURA3 Investigators (2017) Osimertinib or platinum-pemetrexed in EGFR T790M-positive lung cancer. $N$ Engl $J$ Med 376:629-640.

Patel H, Egorin MJ, Remick SC, Mulkerin D, Takimoto CHM, Doroshow JH, Potter D, Ivy SP, Murgo AJ, and Ramanathan RK (2004) Comparison of Child-Pugh (CP) criteria and NCI organ dysfunction working group (NCI-ODWG) criteria for hepatic dysfunction (HD): implications for chemotherapy dosing. J Clin Oncol 22: 6051-6051.

Planchard D, Brown KH, Kim DW, Kim SW, Ohe Y, Felip E, Leese P, Cantarini M, Vishwanathan K, Jänne PA, et al. (2016) Osimertinib Western and Asian clinical pharmacokinetics in patients and healthy volunteers: implications for formulation, 
dose, and dosing frequency in pivotal clinical studies. Cancer Chemother Pharmacol 77:767-776.

Pugh RN, Murray-Lyon IM, Dawson JL, Pietroni MC, and Williams R (1973) Transection of the oesophagus for bleeding oesophageal varices. Br J Surg 60:646-649. Roberts JA, Pea F, and Lipman J (2013) The clinical relevance of plasma protein binding changes. Clin Pharmacokinet 52:1-8.

Socinski MA, Jotte RM, Cappuzzo F, Orlandi F, Stroyakovskiy D, Nogami N, Rodríguez-Abreu D, Moro-Sibilot D, Thomas CA, Barlesi F, et al.; IMpower150 Study Group (2018) Atezolizumab for first-line treatment of metastatic nonsquamous NSCLC. N Engl J Med 378:2288-2301.

Soria JC, Ohe Y, Vansteenkiste J, Reungwetwattana T, Chewaskulyong B, Lee KH, Dechaphunkul A, Imamura F, Nogami N, Kurata T, et al.; FLAURA Investigators (2018) Osimertinib in untreated EGFR-mutated advanced non-small-cell lung cancer. $N$ Engl J Med 378:113-125.

Sticova E and Jirsa M (2013) New insights in bilirubin metabolism and their clinical implications. World J Gastroenterol 19:6398-6407.

Vishwanathan K, Dickinson PA, Bui K, Cassier PA, Greystoke A, Lisbon E, Moreno V, So K, Thomas K, Weilert D, et al. (2018a) The effect of food or omeprazole on the pharmacokinetics of osimertinib in patients with non-small-cell lung cancer and in healthy volunteers. J Clin Pharmacol 58:474-484.
Vishwanathan K, Dickinson PA, So K, Thomas K, Chen YM, De Castro Carpeño J, Dingemans AC, Kim HR, Kim JH, Krebs MG, et al. (2018b) The effect of itraconazole and rifampicin on the pharmacokinetics of osimertinib. Br J Clin Pharmacol 84:1156-1169.

Vishwanathan K, So K, Thomas K, Bramley A, English S, and Collier J (2019) Absolute bioavailability of osimertinib in healthy adults. Clin Pharmacol Drug Dev 8: 198-207.

Vishwanathan K, Yang JC-H, Lee J-S, Krebs M, Dickinson PA, Bui K, Weilert D, So K, Thomas K, and Harvey RD (2016) Effect of itraconazole or rifampicin on the pharmacokinetics (PK) of osimertinib (AZD9291) (Abstract). J Clin Oncol 34 suppl:e14100

Yang JC, Ahn MJ, Kim DW, Ramalingam SS, Sequist LV, Su WC, Kim SW, Kim JH, Planchard D, Felip E, et al. (2017) Osimertinib in pretreated T790M-positive advanced non-small-cell lung cancer: AURA study phase II extension component. $J$ Clin Oncol 35:1288-1296.

Address correspondence to: Karthick Vishwanathan, IMED Biotech Unit, AstraZeneca, 35 Gatehouse Drive, Waltham, MA 02451. E-mail: karthick. vishwanathan@astrazeneca.com 